\title{
Transfusion practice in cardiac surgery: Ars longa, vita brevis, iudicium difficile (the art is long, life is short, and decision difficult)
}

\author{
Giuseppe D'Ancona, MD, PhD
}

\section{See related article on pages 297-302.}

In a retrospective analysis of prospectively collected data presented in this issue of the Journal, Cote and colleagues ${ }^{1}$ tried to determine whether differing practice patterns have an impact on variation in perioperative transfusion rates at a single cardiac surgery center. Of 4823 patients, $40 \%$ received a perioperative transfusion. After adjustment for preoperative and perioperative risk factors, the independent determinants for transfusion rate were year of procedure, surgeon, and anesthesiologist.

I commend Cote and colleagues ${ }^{1}$ for their timely effort in trying to clarify the reasons that may drive transfusions in the perioperative phases of cardiac surgery. In this context, their article is significant to the field and worthy of the reader's attention. In fact, it depicts the standard of practice in many cardiac surgery centers.

The article in many ways emphasizes how, in spite of all our efforts to give a scientific ground to our daily practice, medicine remains for the most part a mixture between art and science. From the article it emerges that, not surprisingly, when no strict intraoperative or perioperative protocols for blood products transfusion or conservation are applied, decisions are based more on personal "gut feelings" than on evidence. Moreover, it is redundant to say that surgical practices are per se heterogeneous, and impact differently on perioperative results, including both perioperative bleeding and transfusion rates. This finding has been already demonstrated in the past by other authors, ${ }^{2}$ and a tremendous effort has been exerted to draw some sort of clinical guidelines ${ }^{3}$ in an attempt to support cardiac surgical practices, rationalize resource management, and minimize risks often associated with inappropriate transfusion of blood products. In spite of that, we have to recognize that there is still lack of high-level evidence to guide blood product transfusion behavior, and most of the recommended decisions are based mainly on expert opinions (class II A

\footnotetext{
From Vivantes Klinikum im Friedrichshain und Am Urban, Berlin, Germany. Disclosures: Author has nothing to disclose with regard to commercial support. Received for publication Sept 17, 2014; accepted for publication Sept 17, 2014; available ahead of print Oct 11, 2014.

Address for reprints: Giuseppe D'Ancona, MD, PhD, Vivantes Klinikum Friedrichshain, Landsberger Allee 49, 10249 Berlin, Germany (E-mail: rgea@hotmail. com).

J Thorac Cardiovasc Surg 2015;149:303-4

$0022-5223 / \$ 36.00$

Copyright (c) 2015 by The American Association for Thoracic Surgery http://dx.doi.org/10.1016/j.jtcvs.2014.09.050
}

level of evidence C). ${ }^{3}$ For example, although there is little scientific base for using a specific hemoglobin level as a trigger for transfusion, this is what we continue to do, accepting, not even in a consistent and standardized fashion, $70 \mathrm{~g} / \mathrm{L}$ of hemoglobin as postoperative cutoff for red blood cells transfusion. In fact, more advanced and "function based" measurements such as oxygen delivery, consumption, extraction rates, and whole-body oxygen-carrying capacity, which would provide a more reliable estimate of the requirement for transfusion, are far from being routinely implemented in daily practice. The issue becomes even more complicated, as shown in the article of Cote and colleagues, ${ }^{1}$ when we are transfusing non-red cell hemostatic blood products without a common cutoff definition for perioperative bleeding and without being guided by point-ofcare tests that assess hemostatic function. ${ }^{1,3}$

I venture to say that restricting the analysis to the "independent determinants" for transfusion will not help us in filling the requirements and is a self-imposed limitation. In my view, transfusion is just an epiphenomenon, the final act of a long chain of previous decisions, either erroneous or correct, in the field of blood conservation and transfusion in which multiple actors are playing a role. In fact, a more holistic approach should be taken. We are coming to different final decisions because we follow different patterns of actions, more or less based on evidence and personal experience. We have to ask ourselves how much of the subjective practice can and should be corrected and guided within the premises of accepted protocols. In this context, a multimodality approach focusing not only on surgeons and anesthesiologists but also on multiple stakeholders, on transfusion algorithms guided by point-of-care testing, and on the many evidence-based and efficacious blood conservation interventions that have been proposed in the literature has already shown to limit blood products transfusion rate while providing adequate hemostasis and optimizing resources allocation. ${ }^{3}$ In spite of these advantages, it is quite evident that only a minority of those involved in the perioperative care of cardiac surgery patients routinely adopt the suggestions derived by the best practices. It is also striking that almost 1 in 2 patients, $40 \%$ in the cohort of Cote and colleagues, ${ }^{1}$ still receive blood products during hospitalization.

In conclusion, cardiac surgery has one of the highest blood products consumption rates relative to other medical practices. Its budgetary burden and intrinsic risk over benefit ratio should be pondered to mandate the development and empower the application of appropriate decision-making processes that regulate institutional blood 
conservation and transfusion policies. At this stage, after accepting the fact that some differences in surgical outcomes will always exist, mainly as a result of subjective skills and attitudes, we should aim at standardizing our strategies to guarantee that outcomes are as uniform and reproducible as possible maintaining affordable costs.

\section{References}

1. Cote C, MacLeod JB, Yip AM, Ouzounian M, Brown CD, Forgie R, et al. Variation in transfusion rates within a single institution: exploring the effect of differing practice patterns on likelihood of blood product transfusion in patients undergoing cardiac surgery. J Thorac Cardiovasc Surg. 2015;149:297-302.

2. Stover EP, Siegel LC, Parks R, Levin J, Body SC, Maddi R, et al. Variability in transfusion practice for coronary artery bypass surgery persists despite national consensus guidelines: a 24-institution study. Institutions of the Multicenter Study of Perioperative Ischemia Research Group. Anesthesiology. 1998;88:327-33.

3. Society of Thoracic Surgeons Blood Conservation Guideline Task Force, Ferraris VA, Ferraris SP, Saha SP, Hessel EA 2nd, Haan CK, Royston BD, et al. Perioperative blood transfusion and blood conservation in cardiac surgery: the Society of Thoracic Surgeons and The Society of Cardiovascular Anesthesiologists clinical practice guideline. Ann Thorac Surg. 2007;83(5 Suppl):S27-86. 